\title{
A Rare Tumor of the Ovary: Carcinosarcoma
}

\author{
Rhizlane Belbaraka ${ }^{\mathrm{a}, \mathrm{b}}$, Amina Taleb ${ }^{\mathrm{a}}$, Hassan Errihani ${ }^{\mathrm{a}}$
}

\begin{abstract}
Malignant mixed mesodermal tumors of the ovary are very rare neoplasms associated with an aggressive clinical course and overall poor prognosis. We report a case of carcinosarcoma of the ovary successfully treated with platinum and ifosfamide and review the literature.
\end{abstract}

Keywords: Tumor; Ovary; Carcinosarcoma

\section{Introduction}

Ovarian carcinosarcomas, also called malignant mixed mullerian tumors (MMMT), is a rare variant of ovarian cancer, accounting for less than $1 \%$ of all ovarian tumors, with fewer than 400 cases reported in the literature [1].

Histologically, carcinosarcoma tumors are composed of both carcinomatous and mesenchymal components, which may be either homologous (composed of tissues normally found in the ovary) or heterologous (containing tissues which are not normally found in the ovary). They are aggressive and have a poor overall survival rate $[2,3]$. We report a case of carcinosarcoma of the ovary successfully treated with platinum and ifosfamide.

\section{Case Report}

A 70-year-old woman who had experienced 2 months of progressive diffuse abdominal pain with vaginal bleeding and

Manuscript accepted for publication July 14, 2010

aNational Institut of Oncology, Rabat, Morocco

bCorresponding author: r_belbaraka@yahoo.fr

doi: $10.4021 / \mathrm{jmc} 1 \mathrm{w}$ abdominal distension presented at our department of medical oncology in the National Institute of Oncology in March 2008. She had no relevant past medical history.

A continuous body weight loss was noted. Upon initial staging, the ECOG performance status was equal to 2. Physical examination showed a pelvic mass. Pelvic ultrasound and computed tomography $(\mathrm{CT})$ revealed a huge and heterogeneous pelvic mass containing solid parts with massive ascites. At laparotomy, the surgeon found a $15 \mathrm{~cm}$ bilateral ovarian tumor densely adherent to the uterus, bladder, peritoneum, and cul-de-sac, and diffuse carcinomatosis. The patient underwent suboptimal debulking surgery including sub-total hysterectomy, bilateral salpingo-oophorectomy, and biopsy of peritoneal node dissection.

The pathologic report was bilateral ovarian MMMT with metastasis to omentum and myometrium. Histologically, the major part was sarcomatous poorly differentiated with the carcinomatous part well differentiated endometrioid adenocarcinoma. Disease stage was IV ovarian MMMT according to the criteria of the international Federation of Gynecology and Obstetrics.

Three weeks after surgery, computed tomography showed multiple nodules of lung and pleural effusion. The serum CA-125 level was elevated (466 U/ml).

The patient accepted first-line chemotherapy with carboplatin AUC 5 and paclitaxel $175 \mathrm{mg} / \mathrm{m}^{2}$ for six courses. After four courses, the lung metastasis showed a partial response (40 per cent) and 60 per cent after achieving six courses with no side effects. No chemotherapy was administrated for consolidation. The treatment was followed by a 9 month progression-free period, and the patient still alive.

\section{Discussion}

Carcinosarcoma is an aggressive tumor, which associates some epithelial elements (carcinoma) with a stromal component (sarcoma). This tumor can be found in the female genital tractus, mostly in the uterus, and it can be found even on the ovaries but it's very rare.

Malignant mixed mesodermal tumors of the ovary are rare neoplasms associated with an aggressive clinical course 
and overall poor prognosis. The results of previous studies have demonstrated that the majority of patients with MMMT of the ovary are Caucasian and their cases are very advanced at the time of surgery [4].

Ovarian MMMT is composed histologically of malignant epithelial and sarcomatous elements, and it is classified to be within homologous or heterologous types according to the origin of its mesenchymal tissue. Homologous MMMT contains malignant stromal elements native to the ovary, whereas heterologous MMMT contains sarcomatous tissue not normally found in the ovary, such as bone or cartilage [5]. In one report, there was no statistical difference in survival between homologous and heterologous MMMT [6].

Research in malignant mixed mesodermal tumors of the uterus have suggested that the sarcomatous and carcinomatous components both arise from a single malignant epithelial precursor which has undergone metaplastic change to a sarcomatous form in some areas of the malignant tissue which contributed to the presence of both histological types [2]. This hypothesis, however, would support the use of regimens active in the treatment of epithelial ovarian cancer, such as platinum. The high rate of recurrence for malignant mixed mesodermal tumors of the ovary treated with platinum-based regimens, however, suggests that this alone is insufficient. Further study in the molecular characteristics of these tumors to guide development of targeted therapies would be of benefit.

Because of the rarity of the disease, no standard treatment has been developed. Both primary cytoreductive surgery and disease stage have been reported as significant prognostic factors $[4,6]$. The role of cytoreduction in epithelial ovarian carcinoma is well established; however, in carcinosarcoma of the ovary, many surgeons doubt about the benefit. The size of residual disease after tumor debulking did not significantly impact survival in a report by Barakat et al [7]. However, Muntz et al reported a benefit of optimal surgical cytoreduction in a review of 27 patients with ovarian carcinosarcoma [6]. He reported a 14\% 2-years survival in suboptimal advanced stage patients compared with $52 \% 2$ years survival in patients achieving optimal cytoreduction. Sood et al reported on 47 patients, and also demonstrated improved outcome with surgical cytoreduction [8]. The median survival for optimal cytoreduction was 25 months compared with 8 months in patients with suboptimal cytoreduction.

Chemotherapy with various regimens has been used in different centers without defined conclusions on efficacy. The Gynecologic Oncology Group (GOG) observed poor response rate and high toxicity with doxorubicin for ovarian MMMT; the authors suggested that it is inadequate as single agent chemotherapy [9]. Several articles have reported cases obtaining long-term survival with different chemotherapy regimens.

There is no consensus on the optimal adjuvant chemotherapeutic regimen in ovarian carcinosarcoma. Multiple chemotherapeutic regimens have been evaluated with modest response rates ranging from $12 \%$ to $100 \%$. The trials are difficult to evaluate due to small numbers of patients, multiple treatment regimens, and occasional use of radiation therapy. The chemotherapeutic regimens most commonly utilized can be simplified into platinum containing regimens compared to non-platinum regimens. A review of the literature does seem to support the use of platinum-based chemotherapy regimens with a $68 \%$ overall response rate in the platinum group compared with $23 \%$ response rate in the non-platinum containing regimens $[10,11]$.

Based on uterine sarcoma data, many institutions combine ifosfamide with platinum, while others follow more traditional epithelial ovarian regimens and incorporate a taxane. The decision to substitute taxol for ifosfamide seems to be based largely on the improved toxicity profile and ease of administration rather than proven efficacy.

A pilot study recently reported by Crotzer safety and activity of ifosfamide and cisplatin [12]. They reported on 8 patients with a median overall survival of 21 months with an acceptable toxicity profile and concluded that this combination warranted further consideration.

In a retrospective review of 28 ovarian MMMT cases treated with platinum and paclitaxel, Duska et al [13] reported 16 patients with complete response and 6 patients with partial response, producing a total response rate of $72 \%$ and overall median survival of 27.1 months.

To summarize, malignant, mixed mullerian tumors of the ovary are very aggressive tumors that were usually diagnosed at an older age compared to women with epithelial ovarian cancer. Similar to this one, MMMT are usually at an advanced stage at the time of diagnosis, and survival after diagnosis varies by stage of disease and histological type. Despite aggressive treatment that includes surgery and chemotherapy, women with these tumors have a significant increased risk of death compared to women with epithelial ovarian cancer and very poor prognosis [14]. The poor prognosis associated with this rare disease emphasizes the need for collaborative prospective studies targeted to better understand the molecular changes of MMMT and the need to design new therapeutic regimens to improve patients' survival.

\section{References}

1. Harris MA, Delap LM, Sengupta PS, Wilkinson PM, Welch RS, Swindell R, Shanks JH, et al. Carcinosarcoma of the ovary. Br J Cancer 2003;88(5):654-657.

2. McCluggage WG. Malignant biphasic uterine tumours: carcinosarcomas or metaplastic carcinomas? J Clin Pathol 2002;55(5):321-325.

3. Brown E, Stewart M, Rye T, Al-Nafussi A, Williams AR, Bradburn M, Smyth J, et al. Carcinosarcoma of the ovary: 19 years of prospective data from a single center. 
Cancer 2004;100(10):2148-2153.

4. Chang J, Sharpe JC, A'Hern RP, Fisher C, Blake P, Shepherd J, Gore ME. Carcinosarcoma of the ovary: incidence, prognosis, treatment and survival of patients. Ann Oncol 1995;6(8):755-758.

5. Boucher D, Tetu B. Morphologic prognostic factors of malignant mixed mullerian tumors of the ovary: a clinicopathologic study of 15 cases. Int J Gynecol Pathol 1994;13(1):22-28.

6. Muntz HG, Jones MA, Goff BA, Fuller AF, Jr., Nikrui N, Rice LW, Tarraza HM. Malignant mixed mullerian tumors of the ovary: experience with surgical cytoreduction and combination chemotherapy. Cancer 1995;76(7):1209-1213.

7. Barakat RR, Rubin SC, Wong G, Saigo PE, Markman M, Hoskins WJ. Mixed mesodermal tumor of the ovary: analysis of prognostic factors in 31 cases. Obstet Gynecol 1992;80(4):660-664.

8. Sood AK, Sorosky JI, Gelder MS, Buller RE, Anderson B, Wilkinson EJ, Benda JA, et al. Primary ovarian sarcoma: analysis of prognostic variables and the role of surgical cytoreduction. Cancer 1998;82(9):1731-1737.

9. Morrow CP, Bundy BN, Hoffman J, Sutton G, Homes- ley H. Adriamycin chemotherapy for malignant mixed mesodermal tumor of the ovary. A Gynecologic Oncology Group Study. Am J Clin Oncol 1986;9(1):24-26.

10. Rutledge TL, Gold MA, McMeekin DS, Huh WK, Powell MA, Lewin SN, Mutch DG, et al. Carcinosarcoma of the ovary-a case series. Gynecol Oncol 2006;100(1):128132.

11. Tate Thigpen J, Blessing JA, DeGeest K, Look KY, Homesley HD. Cisplatin as initial chemotherapy in ovarian carcinosarcomas: a Gynecologic Oncology Group study. Gynecol Oncol 2004;93(2):336-339.

12. Crotzer DR, Wolf JK, Gano JB, Gershenson DM, Levenback C. A pilot study of cisplatin, ifosfamide and mesna in the treatment of malignant mixed mesodermal tumors of the ovary. Gynecol Oncol 2007;105(2):399-403.

13. Duska LR, Garrett A, Eltabbakh GH, Oliva E, Penson R, Fuller AF. Paclitaxel and platinum chemotherapy for malignant mixed mullerian tumors of the ovary. Gynecol Oncol 2002;85(3):459-463.

14. Barnholtz-Sloan JS, Morris R, Malone JM, Jr., Munkarah AR. Survival of women diagnosed with malignant, mixed mullerian tumors of the ovary (OMMMT). Gynecol Oncol 2004;93(2):506-512. 